\title{
HEINRICH GÖDINGS GEDICHT VON HEINRICH DEM LÖWEN.
}

Das sogenannnte volksgedicht von Heinrich dem löwen, das mit den worten beginnt:

Man sagt von starcken helden, sie sein zu preysen hoch;

Darumb so mus ich melden von einem herren such u.s. w.

ist bis in die neueste zeit in einzeldrucken im volke verbreitet worden, ohne dass sich uber den verfasser und die entstehungszeit des werkes bis jetzt sicheres hatte feststellen lassen. Man begnugte sich fast allgemein, dasselbe als eine arbeit des 16. oder 17. jahrhunderts zu bezeichnen, und auch Gödeke meint noch in der zweiten auflage seines Grundrisses zur geschichte der deutschen dichtung b. II, s.321, dass 'die geschichte Heinrichs des löwen ihrer form nach vielleicht erst dem 17. jahrhundert' angehöre. Neu aufgefundene urkundliche zeugnisse liefern uns jetzt uber dichter und entstehungszeit jenes werkes bestimmte nachrichten: wir kōnnen seine abfassung mit sicherheit in das jahr 1585 setzen und dem maler Heinrich Göding in Dresden zuschreiben.

Als herzog Heinrich Julius, sohn des regierenden herzogs Julius zu Braunschweig und Lineburg, mit der prinzessin Dorothea, tochter des kurfursten August von Sachsen, am 26. september 1585 sein beilager feiern wollte, sollten zur erhöhung dieses festes in Wolfenbuttel allerlei ritterspiele, wie ringrennen, balgenstechen und fussturnier, abgehalten werden, zu welchen ausschreiben nach den verschiedensten seiten er- 
gingen. Bei dieser gelegenheit beabsichtigte der junge herzog selbst in zwei kostbaren aufztgen zu erscheinen, nămlich den triumph Heinrichs des löwen und den jagdaufzug der Diana darzustellen. Unter den akten des herzoglichen landeshauptarchives zu Wolfenbuttel, welche sich auf diese festlichkeiten beziehen, hat für uns zunächst wol die erklärung jener 'inventionen' einiges interesse, da sie offenbar von dem fursten selbst herrthit, der sich ja als dichter einer anzahl von deutschen schauspielen wie als begrunder der ersten stehenden deutschen buhne in der geschichte des deutschen dramas und theaters einen nicht unruhmlicheu platz errungen hat. Charakteristisch für die zeit ist besonders die moralische deutung, welche hier sowol die heimische wie die antike sage in einer fur uns uberraschenden weise finden. So wird der sieg, den der herzog Heinrich mit hilfe des löwen uber den drachen davonträgt, in folgender weise ausgelegt.

Interpretatio, M. G. F. undt Hern Invention, nhemblichen des Triumpfs Henrici Leonis.

Obwol S. F. G. sich zu erinnern wissen, das dieser Triumpf undt ganze Historia Hertzog Hein richs des Lewen von dem gemeinem Man also erdichtet, undt ein Fabelwergk ist, dennoch dieweil es von den lieben Altten nicht arg gemeinet, undt auch seine sonderliche bedeutung bat, haben S.F.G. dem gantzen hochloblichem Haus Braunschweigk zue ehren, alss welches von Hochgedachtem Hertzog Heinrichen dem Lewen endtsprossen ist, sich belieben ${ }^{1}$ ) lassen in diesen Triumph auff zu ziben. Es haben aber altte vorstendige leutte Junge Fürsten undt Regenten hirmit underweisen wollen, In was grosse gefahr, Sorg undt Angst, in annhemung des Regiments sie tretten, undt wie sie in demselbigen von allerlei Stinde Laster auch bösen Tirannen undt Underthanen heffitig angefochtten werden, Undt mit denselbigen in teglichen Kanpf liegen müssen, welches alles der streit Heinrici Leonis mit denn Greiffen undt Drachen bezenget undt zu erkennen giebt, undt das es unmilglich ist, das Sie allein aus sich selbst solchen Gewalttigen Feinden wiederstantt thun können. Derwegen lhnen auch hoch von nơten das Sie sich der Tugendt Manheit undt getreuer Leut befleissigen, damit Sie durch derselbigen zuthuendt solchen schrecklichen Feinden nicht allein wiederstehen, besondern dieselbigen auch überwinden undt einen freien Triumpf von Ihrer Uberwindung führen undt haltten milgen, welches alles durch das tugendtriche Manhaffte undt getreue Thir den Leuen, so

1 'bleiben' handschrift. 
Hertzog Heinrichen wieder den Greiffen undt Trachen treulich Beistant gethan, bezeichnet undt angedentet wirt. deutet.

In ähnlicher weise wird auch der aufzug der Diana ge-

Interpretatio der Invention von der Güttin Dianae Ufzug.

Mit dieser Invention wil S. F. G. zu vorstehen geben, das auch Grosse Hern undt Fürsten in Ihrem Mühseligen beschwerlichen Ambt Erliche undt Tugenthaffte Recreationes undt erleuchterung haben müssen, wie man den In den Historien liesset, Sonderlich von dem Cyro undt andern mehr, wie sie sich mit Jagen, Ritter Spiel u. Musiciren undt anderm hinwieder von vielen nohten und sorgen Recreiret undt ermuntert, welches alles die Göttin Diana sambt lhren Nimphis undt underbabenden wilden Leutten andeutet. Es sol aber von grossen Hern bei diesen kurtzweilen die bescheidenheit gehaltten werden, das dadurch die Regierung undt notwendige Ehrengeschefften nicht verabsäumet, und Sie denselbigen in that also nachhengen, das Sie aller Tugendt nndt Vernunfft vorgessen undt daruber fast gahr zu wilden Leutten und Thieren werden, welches uns in dem Acteone dem Jeger, so wegen seines vielen uberflissigen Jagens ghar in einen Hirs (wie die Poeten tabuliren) verwandelt undt verflucht, ohne $\mathrm{Zweifel}$ furgehalten wirdet.

Auch ther die veranstaltung der aufztige sind wir näher unterrichtet. Der triumphzug Heinrichs des löwen wurde durch zwei herolde eröffnet; ihnen folgten vier 'personen so Trachenheubtter vndt Greiffenklauen tragen', dann sechs 'welsche Musikanten', ein geschmlickter 'Spiessjunge' einen schild tragend, der als wappen das weisse ross zeigte, und zuletzt der berzog selbst auf dem triumphwagen, hinter welchem vier geschmuckte hengste gefuhrt wurden. In ăhnlicher weise war in jenem jagdzuge der herzog als Diana von musikanten, wilden männern, nymphen, dem Aktäon u. s. w. umgeben.

Diese darstellung Heinrichs des löwen von seiten des herzogs Heinrich Julius, von welchem sich die kunde naturlich auch an dem wohnorte der braut, in Dresden, verbreitete, ist offenbar die veranlassung gewesen, dass sich von hier aus am 13. september 1585 ein maler Heinrich Göding an den vater des bräutigams, herzog Julius, mit einem schreiben wante, das sich zwischen den oben erwähnten akten erhalten hat. Er erbietet sich in diesem ein von ihm gefertigtes gedicht, das die wunderbaren schicksale Heinrichs des löwen behandelt, zu ehren der nahe bevorstehenden hochzeit mit kupfern zu schmucken. 
Ein exemplar des druckes jenes gedichtes, das er dem briefe beigelegt hatte, liegt ebenfalls noch in jenen akten. Das schriftstuck selbst lautet folgendermassen:

Durchlauchtigster Hochgeborner Furst, gnedigster Furst vnnd Herr. Ewer furstliche Gnadenn seinn Meine vnnterthenige willige Diennste inn trewen befornn. Gnedigster furst unnd Herr, Nach dem ich Offter gehörtt vonn der schünenn Historienn, so Ewer furstliehe gnadenn forfahrenn allss einn kunner vnnd tewer heltt sol ihnn grosser gefahr geleistet habbenn, auch die warzeichenn, so $\mathrm{zw}$ braunschweigk ihnn Duhme noch gesehen werden, anss weissenn vnnd weill, gnediger furst vnnd herr, fihl geschichte vnnd dattenn beschriebenn werdenn, welches denn hohenn Pottentattenn Nachkommen rumlich, vnnd ess nicht auss der acht kommen möchte, wie mann dann vonn anndern kunnen heldenn schöne geschicht vnnd tattenn lissett, alss vonn dem Amadis anss Frannckreich vnnd anndern fihl mehr, wie Ewer furstliche gnadenn gnedigst wissen tragenn, auch der Chrrfurst $2 w$ Sachssenn meinn gnedigster Herr solches und andere mer mitt Fleiss lassenn mahlen,1) wie $\mathrm{zw}$ ersehenn. Derwegenn Ewer fürstliche gnadenn vnnd dissenn löplichenn fürstlichenn stam $\mathrm{zW}$ ehrenn ich diss gedicht inn eull gemacht ${ }^{2}$ ), vnnd op mir solchess geschichte fast auss gefallenn vnnd aller dinnge nicht recht in der eulle geordnett, so wollenu doch ewer furstliche gnaden gnedigst vonn mir diesses gerinngess kleinness wergk in gnadenn aufnemen, vnnd nach der selben gnedigenn meinung, da einn Manngel darinne gefundenn, durch anndere verstenndige bessernn lassenn, denn ess jha schade wehre, dass solche schöne historie sol gar vnntergehenn vnnd ennttlich vergessenn werdenn. Vnnd weil, gnediger furst vnnd Herr, fihl schönner historienn gemahlett vnnd ihnn kopfer gestochen werdenn,

1) An des briefschreibers bertihmtes werk 'Ausszug der Eltisten vnd fürnembsten Historien des vralten streitbarn vnd beruffenen Volcks der Sachssen | Insonderbeit aber des Keyserlichen Koniglichen Chur- vnd Fürstlichen stammes der Gros vnd Herzogen zu Sachssen...' (Dressden 1597 u. 1595) darf man hier schwerlich denken, da er mit dieser arbeit, wie Andresen (Der deutsche Peintre Graveur) s. 75 berichtet, 'nachdem er sehr viele werke für das churhaus Sachsen in langer zeit ausgeftihrt, in seinem alter sich noch ein gedächtnis habe stiften wollen'. Will man die worte auf Göding selbst beziehen, so könnte man eher an die bildnisse sächsischer fürsten in der gewehrgallerie zu Dresden denken (mitteilungen des kgl. sächs. vereins für erforschung und erhaltung der vaterländischen altertïmer, 3. heft, Dresden 1846, s. 43); vielleicht beziehen sie sich auch auf uns ganz unbekannte werke.

2) Vergleiche den letzten vers des gedichts nach dem ursprüng. lichen drucke:

Von wegen der Geschichten hab ich dis Lied gedicht,

In eyle thet ichs machen, hets sonst besser gericht,

Dem Fürsten Stamm zu Ehren u. s. w. 
anndern pottentattenn $\mathrm{zW}$ ehren vnnd gedechtnuss, binn ich offter willennss gewessenn, $\mathrm{zw}$ ehrenn denn braunschweigischenn vnnd lineburgischen furstlichenn stam solches $\mathrm{zW}$ ehrenn auch aufs kopfer $\mathrm{zW}$ bringenn, abber biss daher nicht weilligk gewessenn. Mich auch verursacht disse historie Inventsion vnnd Triumpf ${ }^{1}$ ) Ewer furstliche Gnadenn fiel geliptenn sohnn Herzog Henrich Julliuss zw ehrenn auf diss beilager willich vnnd vnnderthenig $\mathrm{zw}$ ordnenn, vnnd binn ferner Ewer furstliche gnaden vnnderthenig nach meinenn Vermögenn inn mehren $\mathrm{zw}$ dienenn ganncz willigk vnnd thun ewer furstliche gnadenn sampt der selbenn fiel geliptenn gemahl sampt der jungenn herschaft vnd frewlleinn in schucz dess hochstenn bevehlen.

Dattum Dresdenn denn 13 September Anno 85

Ewer fürstliche gnaden

unntertheniger vond

gehorsammer

Henrich götting

Mabller.

Auf der ruckseite des briefes ist von der hand des herzoglichen sekretärs das präsentatum mit: 'Wolffenbuttel 23 7bris Ao 85' vermerkt worden. Ferner: 'Heinrich Gotting Mahler zu Dresden habe den Triumphum Henrici Leonis gemahlen ${ }^{2}$, schicke Illustrissimo ein Exemplar, wils in Kupffer stechen lassen'. Der entwurf einer antwort ist leider nicht erbalten; auch jede darauf zielende andeutung fehlt. Wir können daher nicht mit bestimmtheit sagen, ob herzog Julius auf den vorschlag eingegangen ist oder nicht. Da sich jedoch keine kunde von einer solchen bildergeschmulckten ausgabe des gedichtes erhalten hat, so ist es wahrscheinlich, dass der furst von des malers anerbieten keinen gebrauch gemacht hat.

Das kann uns nicht wunder nehmen. Julius war ein sparsamer haushalter, dessen nllchterner sinn mehr auf praktische ziele als auf die förderung der kunst gerichtet war, welcher im gegensatz $\mathrm{zu}$ ihm sein sohn Heinrich Julius eine verständnisvolle, freigebige pflege zuwante. Möglich, dass bei Julius auch politische uberzeugungen mitwirkten. Er war keineswegs ein verehrer der politik seines grossen alnen. Streng kaiserlich gesinnt erblickte er vielmebr in dem bestreben Heinrichs der reichsgewalt sich gleichzustellen einen offenbaren verrat,

1) Vgl. dieselben ausdrücke s. 279. Man sieht hieraus, dass dem dichter die geplanten veranstaltungen in Wolfenbittel wolbekannt waren.

2) So wol fälschlich statt 'gedichtet' geschrieben, 
der seinem hause zum grōssten nachteile ausgeschlagen sei. In seinem testamente vom 29. juni 1582 stellt or daber seinea söhnen 'zu steter warnung und abscheu' das schicksal ihres ahnherrn warnend vor augen. ${ }^{\text {) }}$

Die vermăhlung eines braunschweigischen herzogs mit einer kursächsischen prinzessin war für Göding in doppelter hinsícht eine willkommene veranlassung seine dienstfertigkeit $z u$ bezeugen. Nach beiden seiten hatte or naturliche beziehungen: seiner geburt nach gehörte er den braunschweigischen, seiner dienstlichen stellung nach den sächsischen landen an. In der geschichte der deutschen kunst ist der name $\mathrm{H}$. Gödings längst ein bekannter.') Die form des namens ist sowol bei ihm selbst als auch bei seinen zeitgenossen eine verschiedene gewesen; es begegnet neben Göding auch Götting, Gödig, Godeg u. a. ${ }^{3}$ ) Da aber in der kunstgeschichte der name Göding sich immer mehr einzuburgern scheint, so habe ich mich, um verwechselungen zu vermeiden, dieser bezeichnung hier ebenfalls anschliessen zu mulssen geglaubt, obwol die unterschrift des obigen briefes fur die form Götting spräche.

1) Vgl. Rehtmeier's braunschw.-lüneb. chronik (Braunschweig, 1722) 8. 1038 .

2) Vgl. über Göding sowie über seine gemälde und kupferstiche Ch. Schuchardt in R. Naumanns Archiv f. d. zeichn. klinste (Lpzg. 1855) b. I, 94-101. - Julius Hübner im Archiv f. d. sächs. geschichte, hg. von W. Wachsmnth und K. v. Weber, b. II, 8. 184. - G. K. Nagler, Die Monogrammisten b. IIl (Munchen, 1863) s. 337-339. - J. D. Passarant, Le Peintre Graveur b. IV (Leipsic, 1863) s. 232-235. - Andreas Andresen, Der deutsche Peintra=Graveur oder die dentschen maler als kupferstecher, b. I (Leipzig, 1864) s. 71--98. - C. Clanss in der Allgem. deutschen biographie, b. IX, 8. 319. Eine eingehende arbeit über Godings kunsttätigkeit auf grund archivalischer forschungen steht von herrn Dr. Berling in Dresden zu erwarten. Dieselbe wird im Neuen archiv für sächsische geschichte, jahrg. 1887, heft 3 a. 4 erscheinen.

s) Auf dem titel seines werkes: 'Ausszug der Eltisten und furnembsten Historien des - Volcks der Sachssen etc.' wird als des verfassers 'Heinrich Godegen von Braunschweig' gedacht, während die unterschrift der widmung lautet: 'Dreszden den 5. May jm 1597. Jahr E. F. G. Unterthenigster Gehorsamer Heinrich Göding von Braunschweig'. Auf dem titel des zweiten teiles des werkes begegnen wir ebenfalls wider der form 'Godegen' (nach freundlicher benachrichtigung des berrn bibliothekars Dr. Schnorr v. Carolsfeld in Dresden). 
Heinrich Göding ist, da er am 28. april 1606 in einem alter von 75 jahren in Dresden verstorben ist ${ }^{1}$ ), zu ende des jahres 1530 oder zu anfang des folgenden in Braunschweig geboren. Um das jahr $1557 \mathrm{kam}$ er nach Dresden und hier hat er als hofmaler im dienste der kurfursten August, Christian I. und Christian II. eine rege wirksamkeit entfaltet. Er wird ein freund des jungern Lucas Cranach genannt. Neben der malerei betrieb er auch den kupferstich. In beiden zeigt er sich nach den urteilen der kunstkenner 'als ein technisch gewanter kunstler, der sich jedoch nicht ther die manirierte und handwerksmässige kunstweise seiner zeit erbob'.2)

Dass der verfasser jenes gedichts mit diesem maler eine person ist, muss schon nach dem oben mitgeteilten briefe als ausgemacht gelten. Eine weitere bestätigung erbalten wir aus dem titelblatte des druckes, der jenem schreiben beilag. Dasselbe lautet:

Eine schöne alte Histori | von einem Fürsten vnd Herrn, Herrn |

Hertzogen zu Braunschweig vnd Lüne-/burgk: In gesangs weis gerichtet, | Im 1585. Jahr. | H. G.

Die buchstaben H. G., welche den dichter andeuten sollen, entsprechen ganz dem monogramme des malers Göding, welches ebenfalls ein H. G., H. G. B. (d. i. Heinr. Gōding Brunsvicensis) oder ăhnliches enthält. ${ }^{3}$ ) Für seine braunschweigische abstammung sprechen unverkennbar auch die worte des letzten verses seiner dichtung, in denen er sagt, dass er dieselbe dem furstenstamme und seinem 'vaterlande' $\mathrm{zu}$ ehren angefertigt habe.

Der druck des gedichts besteht aus 16 blättern in quart mit den signaturen $A_{11}-D_{11}$. Die beiden letzten blätter sind leer, doch ist die vorderseite des vorletzten blattes mit recht geschmackvollen ornamenten angefullt, in denen wir vielleicht proben der kunstfertigkeit des dichters selbst zu erkennen haben. Die verse sind fortlaufend wie prosa gedruckt; jedes reimwort ist durch einen dahinter gesetzten strich bezeichnet. Die ein-

1) Michaelis Inscriptiones der grabmonumente aus der kirche und vom kirchbofe der frauenkirche in Dresden, 1714, s. 97 (nach einer mitteilung des herrn Berling).

2) Vgl. C. Clauss in der Allgem. deutschen biographie b. X, s. 319.

3) Vgl. Nagler a. a. 0. s. 337 ff.; Andresen a. a. 0. s. 71, 93, 94 u. 8. w. 
zelnen strophen sind abgesetzt. Auf jeder seite stehen vier strophen, das ganze gedicht enthält deren 104. Ueber drucker und druckort des buches, die in demselben nicht genannt werden, habe ich nichts in erfahrung bringen können. Die ausgabe ist, wenn nicht ein unicum, so doch jedenfalls sehr selten. Sie ist in der betreffenden literatur meines wissens bis jetzt niemals erwähnt worden; in keiner der vielen bibliotheken, bei welchen ich mich nach ihr erkundigt habe, war sie vorhanden.

Das werk ist in dem sogenannten Hildebrandstone gedichtet, in dem ja die meisten volkstumlichen dichtungen der zeit verfasst worden sind. Die darstellung des dichters ist gewant, seine sprache leicht und fliessend. Dass er den echten volkston richtig $\mathrm{zu}$ treffen verstand, bezeugt das lange, ununterbrochene leben, das sein gedicht bis in unser jahrhundert hinein in der volksliteratur gefuhrt hat. Eine weitere bedeutung des gedichts besteht darin, dass es uns einen alten schon ofter behandelten sagenstoff in neuer und zwar derjenigen fassung uberliefert, welche fur die folgenden jahrhunderte die massgebende geblieben ist. Mit den fruheren verwanten dichtungen 1), wie der Michael Wyssenheres ${ }^{2}$, hat Gödings werk auch das gemein, dass der name des helden nirgends genannt wird. Er heisst im titel ganz allgemein nur herzog zu Braunschweig und Lüneburg, im texte des gedichts fast durchgehend nur der herr. ${ }^{3}$ ) Denn die vermutung Bartschs ${ }^{4}$ ), dass die letzte

1) Vgl. tiber diese im allgemeinen die einleitung Bartschs zu seiner ausgabe des Herzogs Ernst (Wien, 1869), über die sage den aufsatz W. Muillers 'Die fahrt in den osten' in Schambachs und Müliers Niedersächsischen sagen und märchen (Göttingen, 1855), s. 389 ff. Schon bald nach seinem tode batten sich lied und sage der gestalt Heinrichs des löwen bemächtigt. Vgl. Germania, hg. von Bartsch; neue reihe, XIX. (XXXI.) jahrg., 8. $151 \mathrm{ff}$.

2) Vgl. den abdruck in Massmanns Denkmälern deutscher sprache und literatur (München, 1828) s. 123-137.

3) So strophe $1^{2} \cdot 5^{2} \cdot 6^{3} \cdot 9^{4} \cdot 11^{2} \cdot 11^{4} \cdot 12^{2} \cdot 12^{4} \cdot 13^{2} \cdot 14^{1} \cdot 15^{4} \cdot 16^{2} \cdot 16^{4}$. $17^{2} \cdot 18^{1} \cdot 19^{2}$ und so fort. Daneben wird er dann aber auch 'der Landesherr' 881, 'der fromme Landesherr' 92. 652 , 'der Herr zu Braunschweig' $95^{3}$, 'ein Herre von Braunschweig hochgeborn' $36^{3}$, 'der hochgeborne Fürst' $3^{1}$, 'der werde Mann' $13^{3}$. 231', 'der thewre Held' 15', 'Hertzog' $71^{3}$, 'Hertzog von Braunschweig' 594 und ähnlich genannt.

4) Vgl. Herzog Ernst, hg. v. K. Bartsch (Wien, 1869) в. CXXI, anm. 2. 
strophe, welche den namen Heinrichs nennt, von einem erneuerer herruhre, bestätigt sich in der tat. $Z$ war hat die änderung nicht Simrock gemacht, wie Bartsch mutmasst, sondern schon ein fruherer bearbeiter, der uberhaupt die letzten beiden strophen vollständig umgestaltet bat. ${ }^{1}$ )

Welchen quellen Göding bei seinem werke gefolgt sei, entzieht sich unserer näheren beurteilung. Auf die abweichungen seiner darstellung von der Michael Wyssenheres hat schon Bartsch aufmerksam gemacht ${ }^{2}$ ) und dabei mit recht hervorgehoben, dass man für dieses gedicht eine andere quelle annehmen musse. Ich halte die annahme mundlicher uberlieferungen bei Gōding für vollkommen ausreichend. Dass damals die sage von dem löwenherzoge in Braunschweig noch vollkommen im schwange war, beweist u. a. das oben mitgeteilte zeugniss des herzogs Heinrich Julius. Es ist selbstverständlich, dass dieselbe unserm dichter als geborenem Braunschweiger ebenfalls wol vertraut war. Viele denkzeichen in der stadt und vor allem im alten Blasiusdome erinnerten zu der zeit in noch grösserer anzahl als jetzt an die wunderbaren abenteuer des herzogs im fernen morgenlande und an den löwen, den treuen gefährten des fursten. ${ }^{3}$ ) Der dichter fuhrt selbst deren an: den löwenstein auf dem burgplatze, den man noch heute als das wahrzeichen der stadt und ibres welfischen furstenbauses betrachtet (str. 1013), das grabmal des herzogs im dome (str. 994 und 1024), die geierkralle, welche noch zu Rehtmeiers zeit (1707) uber dem grabe Heinrichs von dem gewölbe der kirche herabhing (st. $19^{3}$ und 1021).4) Dass diese erinnerungen und die kenntuiss braunschweigischer örtlichkeiten

1) Vgl. den abdrack derselben s. 289.

2) A. 2. o. s. CXXII.

3) Vgl. den schönen aufsatz L. C. Bethmanns die gründung Braunschweigs und der dom Heinrichs des löwen in Westermanns monatsheften ang. 1861 s. 525 ff., insbesondere s. $556 \mathrm{ff}$.

- Vgl. Rehtmeyer der stadt Braunschweig kirchen-bistorie. I. teil. (Braunschweig, 1707) 8. 103. Später wurde die klaue dort entfernt und in der sakristei der kirche verwahrt, wie ebenfalls Rehtmeyer a. a. o. in dem 1720 erschienenen V. t. Suppl. s. 36 berichtet. Die sog. geierkralle ist tibrigens nicht, wie man nach Bethmanns angabe a. a. 0. 8. 558 annehmen könnte, verloren gegangen, sondern wird noch jetzt in dem dome gezeigt. 
wie des giersberges, den er auffallender weise in 'Geyersberg' verhochdeutscht (str. $37^{3}, 43^{3}$ ) dem.dichter vollkommen geläufig blieben, kaun uns bei seiner abstammung nicht wunder nehmen. Es bestärkt uns dies in der annahme, dass er uns die sage von Heinrich dem löwen im wesentlichen in der form uberliefert hat, in welcher sie damals im munde des volkes lebendig war.

Als älteste form unseres gedichts hat man in neuerer zeit eine handschrift angesehen, die sich in der herzoglichen bibliothek zu Wolfenbuttel befindet (207,5 Extr. $4^{0}$ bl. 27-34). ${ }^{1}$ Es ist dies aber ganz offenbar nur eine abschrift unseres druckes, die nach dem charakter der schrift zu urteilen in der zweiten hälfte des 17. jabrhunderts gemacht wurde. Dass dieselbe in Braunschweig angefertigt sei, dafür spricht die beibehaltung der richtigen, noch jetzt gebräuchlichen namensform 'Giersberg' statt der im druck befindlichen 'Geyersberg' (str. $37^{3}$ und $43^{3}$ ). Der titel hat den zusatz 'Hinrick de Lauwe geheten', aber, wie schon Pröhle nach einer mitteilung dr. Milchsacks angibt ${ }^{2}$, von einer jungeren hand hinzugefugt. Die absehrift scheint zwar nach dem originaldrucke selbst gemacht zu sein, ist aber im ganzen ziemlich fluchtig angefertigt. Es finden sich in ihr nicht wenige abweichungen, die meistens auf einsetzung jungerer wortformen, wie 'ihnen' fur 'ihn' (eis), 'haben' fur 'han' (habere) 'vollbracht' fur 'verbracht' (str. 404) u. a., z. t. aber auch auf scbreibfehler und missverständnisse des abschreibers hinauslaufen. Die strophenzahl ist die alte geblieben (104), obwohl, nur 103 gezäblt werden; denn unter 46 stehen zwei strophen.

1 So $\mathrm{H}$. Pröhle in der 2. auf. seiner deutschen sagen (Berlin, 1879) s. 290. Die handschrift wird in v. Praun's Bibliotheca Brunsvico-Luneburgensis (Wolfenb. 1744) 8. 61 str. 232 und in A. U. Erath's Conspectus historiae Brunsvico-Luneburgicae (Braunschweig, 1745) 8. 51 str. 1727 als in der Wolfenbuittler bibliothek befindlich angeführt. E.J. Koch sagte in der 2. aufl. seines compendiums der deutschen literaturgeschichte (Berlin, 1795) b. I 8.134, dass sie dort nicht aufzufinden gewesen sei. Es erleidet keinen zweifel, dass unter jener handschrift die von Praun, Erath u. 8. w. erwähnte zu verstehen sei. Ausser jenem gedicht enthält die handschrift noch sehr verschiedenartige dinge, die in sehr verschiedenen zeiten geschrieben sind.

Pröhle a. a. 0. 8. 290. 
Widerholungen wird der druck nicht wenige gebabt haben. Aber es liegt in dem schicksale aller derartigen dichtungen, die auf schlechtes löschpapier gedruckt, von den niederen volksschichten auf jahrmärkten und strassen gekauft, von den gebildeten kreisen aber jahrbunderte lang vornehm missachtet wurden, dass die meisten drucke fast durchgebends kein langes leben gefristet haben werden. Sio wurden in den bibliotheken verschmäht, und so finden wir denn auch jetzt in ihnen, wie mich reichliche anfragen gelehrt haben, derartige drucke verhältnissigmässig nur äusserst selten.

Der erste druck, der mir nach der ausgabe von 1585 begegnet ist, stammt aus dem jahre 1727; er befindet sich in der königlichen bibliothek zu Berlin, in welche er aus der von Meusebachschen buchersammlung gekommen ist. Der titel lautet folgendermassen:

Wahrhafftige | Beschreibung | Von dem grossen Helden | und Hertzogen | BEINRICH | dem Löwen, und seiner wunderbaren höchst- $\mid$ gefährlichen Reise. | [bolzschnitt darstellend einen mann in kniehosen und langeun rocke, daneben einen löwen mit erhobenen vordertatzen, im hintergrunde eine barg] | Auf Begehren vieler Liebhaber itzo wie-|der aufs neue aufgelegt. (3) | Braunschweig und Leipzig. 1727.

$20 \mathrm{bl}$. in $8^{\circ}$. sign. $\mathrm{A}_{2}-\mathrm{C}_{3}$.

Auf der rlickseite des titelblattes und auf der folgenden scite steht eine kurze, sehr luckenhafte ubersicht der braunschweigischen berzōge von Heinrich dem löwen bis auf Anton Ulrich. Auf s. 4 und 5 folgt eine 'Vorrede. An den glinstigen Leser', s. 7-16 eine prosaische erzăhlung des lebens und der taten Heinrichs des löwen. Auf s. 16 beginnt auch noch der abdruck des gedichts, der bis 8.36 reicht. Den schluss des buches nehmen zwei erzählungen von treuen löwen aus $\mathrm{Di}$ dacus Apolephtes Lusitanus' historischen erquickstunden und Caspar Titius' theologischem exempelbuche ein.

Dass diese zusammenstellung des buches wirklich aus der auf dem titel angegebenen zeit stammt (1727), geht aus der erwăhnung des löwendenkmals auf dem burgplatze zu Braunschweig hervor. Es wird gesagt, dass dieses 'nur noch vor wenig jahren in etwas erböhet, auch der pfeiler gåntzlich renoviret und abgeputzet worden.' Da einige seiten vorher der im j. 1704 erfolgte tod herzog Rudolf Augusts angefuhrt wird, so 
kann es sich nur um die restauration des denkmals im j. 1721 handeln, von welcher Rebtmeier in seiner braunschweig-luneburgischen $\cdot$ chronik s. 1585 spricht.

$\mathrm{Ob}$ dem herausgeber bei seiner arbeit das gedicht in dem ursprünglichen drucke vorgelegen hat oder ob wir in der uberlieferung zwischenglieder anzunehmen haben, lässt sich, da uns letztere vorläufig fehlen, mit sicherheit nicht feststellen. Manche veränderungen sind in dem gedichte vorgenommen. Wir finden ältere wortformen mit jungeren vertauscht, wie 'greif' mit 'griff' (str. 172), 'schneit' mit 'schnit' (str. 173), 'steig' mit 'stieg' (str. 191), 'schrei' mit 'schrie' (str. 234), sodann ungebräuchlich gewordene ausdrucke durch neuere ersetzt, wie 'wirtschaft' durch 'beilager' und 'hochzeit' (str. 333. 404. 784. 893. 931. 941), 'hort' durch 'schiff' (str. 283) u. 8. w. Aus dem 'drachen' ist ein 'lindwurm' (str. $20 \mathrm{ff}$.), aus dem 'greif' ein 'vogel greife' (str. 16'), aus dem 'haus' ein 'schloss' (str. 601), aus dem 'wirth aussm nobiskrug' oder dem 'nobiswirth' der 'teufel' oder 'satan' (str. $32^{2}$ und 44') geworden; fur 'das orth' ist 'der orth' gesagt (str. 38') u. s. w. Mitunter greift eines solchen unbekannteren wortes willen die veränderung weiter. So ist z. b. 'er war ein Degen hart' in 'er war von edler Art' (str. 24), 'nach der Burgk war jhm gach' in 'nach der Burg war sein gang' (str. 483) verwandelt. Oft liegen auch offenbare fluchtigkeitsfehler und missverständnisse zu grunde, wie str. 124, wo die worte: 'ich thue solchs nicht meim Herrn' zu 'ich thue solchs nicht, mein Herr' umgestaltet sind. Ganz umgearbeitet sind die beiden letzten strophen, welche in dieser ausgabe folgendermassen lauten:

Ach GOtt du wollest behüten diss hohe Fürsten-Hanss,

In aller Regenten Zeiten theilen den Seegen aus.

Auch gn:̈diglich bewabre für Pest, Krieg, Raub und Brandt

Und gnädiglichen mehre die Nahrung in dem Land.

Zum stetigen Andencken dieser wunderbaren.Geschicht

Und auch zu ewigen Ehren des Herren Hertzog Heinrich

Und seinen getreuen Löwen ist dieses gantze Gedicht

Dem Fürstl. Stamm zu Ehren in Braunschweig aufgericht.

Die zahl der strophen ist die alte geblieben. Sie sind im drucke abgesetzt; doch sind innerhalb derselben die verse fortlaufend wie prosa gedruckt. Der text wird durch drei kunst- 
lose holzschnitte unterbrochen, von denen zwei ganz gleich sind. Diese stellen ein schiff auf dem meere dar, der dritte die uberfuhrung des löwen nach Braunschweig.

Die heimat dieses bearbeiters haben wir höchst wahrscheinlich in Braunschweig zu suchen. Abgesehen davon dass er in seiner einleitung eine ziemliche bekanntschaft mit der braunschweigischen geschichte zeigt, verrăt er auch in dem gedichte selbst volle vertrautheit mit der stadt Braunschweig. Denn ganz richtig schreibt er str. $37^{3}$ und $43^{3}$ statt der hochdeutschen form 'geyersberg' die noch heute ublicbe niederdeutsche 'giersberg', und ebenso setzt er str. $48^{3}$ statt der allgemeinen bezeichnung des 'hauses' des herzogs den namen 'mosthauss' ein, mit welchem damals das môshâs, der alte saalbau Heinrichs des löwen, bezeichnet wurde, der nach langer verwahrlosung in unseren tagen zu neuem glanze erstebt. Den vers (str. 102'): 'Ein Greiffenklaw thut hangen vber dieses Fursten Grab' verwandelt er in 'Eine Greiffen-Klaue auch hanget zu Braunschweig in den Duhm', offenbar weil er weiss, dass die klaue, die noch 1707 uber dem grabe des fursten hing, inzwischen dort entfernt und in der sakristei der kirche untergebracht war. ${ }^{1}$ Auch der schluss des werkes spricht fur die braunschweigische heimat des bearbeiters, da er hier (str. 104) von der ursprunglichen fassung stark abweichend sagt, es sei 'dieses gantze Gedicht dem Furstl. Stamm zu Ehren in Braunschweig aufgericht.' Bei Göding findet sich ein derartiger binweis nicht; wir haben daher in diesen worten wol eine änderung zu sehen, die durch persönliche verhältnisse des herausgebers selbst veranlasst ist.

Die ausgabe von 1727 ist im 18. und 19. jahrhundert,widerholt nachgedruckt worden; sie ist die allgemein verbreitete form der sog. jahrmarktsausgabe geblieben. Ich kann vier verschiedene drucke der art nachweisen, deren titel folgendermassen lauten.

1. Wahrhafte Beschreibung | von dem grossen | Helden und Herzogen | HEINRICH | den Löwen, | und seiner | wunderbaren höchstgefährlichen Reise. | [bolzschnitt, darstellend vorn 6 krieger za pferde und zu fuss, dahinter ein zeltlager und drei grosse beerhaufen]|

1 Vgl. die anmerkung 4 auf s. 286. 
Auf Begehren vieler Liebhaber aufs neue aufgelegt. $\mid$ Braunschweig und Leipzig. ( 3 '.

$20 \mathrm{bl}$. in $8^{0}$. Sig. $A_{2}-C_{g}$. Seitenzahlen fehlen:

2. Wahrhafte Beschreibung | von dem grossen | Helden und Herzogen | Heinrich | dem Löwen, | und seiner | wunderbaren höchstgefährlichen Reise. |[holzschnitt wie der in no. 1] | Auf Begehren vieler Liebhaber aufs neue aufgelegt. | Brannschweig und Leipzig. (3.

$20 \mathrm{bl}$. in $8^{\circ}$. Sign. $A_{2}-C_{3}$. Seitenzahlen $4-40$.

3. Beschreibung | von dem grossen | Helden und Herzogen | Heinrich dem Löwen, | und | Seiner wunderbaren höchstgefährlichen | Reise. | [holzschnitt wie der in no. 1] | Ganz neu gedruckt. 3.

20 bl. in $8^{\circ}$. Sign. $A_{2}-C_{3}$. Seitenzahlen $4-40$.

4. Beschreibung | von dem grossen | Helden und Herzogen | Heinrich dem Löwen, | and | seiner wunderbaren und höchstgefährlichen | Reise. | [holzschnitt, welcher vorn einen reiter mit gezogenem schwerte, dahinter 2 abteilungen fussvolk mit je 2 fahnen und an der linken seite die ecke eines grossen gebäudes zeigt.]|Ganz neu gedruckt. 3 .

16 bl. in $8^{\circ}$. Sign. $A_{2}-B_{2}$. Seitenzahlen 6-32.

Die drei ersten drucke befinden sich im besitze der $\mathrm{kgl}$. bibliothek zu Berlin, der vierte in dem des verfassers. Gewiss würde sich ihre zahl noch sehr vermehren lassen, wenn ihre erhaltung durch die ungunst der verhăltnisse nicht 80 stark beeinträchtigt wäre.

Alle vier drucke weichen darin von ihrer vorlage ab, dass sie die drei teile der einleitung umgestellt haben. Sie bringen zuerst die 'Vorrede an den gunstigen Leser', dann die ubersicht uber die braunschw. herzogge und darauf die prosaische erzählung von Heinrichs leben und taten. Es folgt sodann, wie dort, das gedicht selbst mit abgesetzten strophen und fortgedruckten versen und zuletzt der anhang. Ausserdem haben sie der ausgabe von 1727 gegentuber eine anzahl grober druckfehler gemeinsam. Sie nennen alle vier den herzog Heinrich Julius 'Heinrich Tulius', als dessen geburtstag statt des 15. den 5. october; sie setzen den tod herzog Friedrich Ulrichs statt in das jahr 1634 in d. j. 1643, die geburt herzog Augusts statt in d. j. 1579 in 1597 u. a. m. Auch einige kurzungen in der vorrede finden sich bei ihnen in gleicher weise. Ebenso weichen sie in der widergabe des gedichts von der ursprünglichen quelle nicht selten gleichmässig $a b$, während sich zwischen ihnen selbst verhältnissmässig wenige verschieden-

Beitrăgo zur geschichte der deutschen sprache. XIII. 
heiten zeigen. Es würde hier zu weit fuhren im einzelnen darauf einzugehen.

Ebenfalls auf jener zusammenstellung beruht trotz zahlreicher und weitgehender abweichungen die prosaauflösung des gedichts, von der mir zwei drucke bekannt sind. Der titel des ersten, welchen mir herr professor dr. Steinacker in Braunschweig freundlichst zur verfügung stellte, lautet:

Merkwürdige | Beschreibung von dem Leben | des grossen | Herzogs und Helden | Heinrich des Löwen, | und von | seiner höchstgetährlichen Reise. | [holzschnitt, darstellend einen ritter mit einem lowen den weg nach einem tore hinauf schreitend] / Frankfurt und Leipzig. 3.)

20 bl. in $8^{\circ}$. Sign. $A_{2}-C$. Seitenzahlen 4-40.

Der titel des zweiten druckes, den mir herr oberlebrer dr. Pröhle in Berlin zur benutzung ubersandte, ist folgender:

Leben und Thaten | des grossen Helden | Heinrich des Löwen, | Herzog zu Braunschweig. | [Holzschnitt wie der des ersten druckes] Einbeck, | bei H. Ehlers.

$18 \mathrm{bl}$. in $8^{\circ}$. Sign. $A_{2}-C$. Seitenzahlen 4-36.

Beide drucke können frubstens aus dem anfange des 19. jahrhunderts stammen, da auf dem burgplatze, welchen in beiden auf s. 3 ein holzschnitt darstellt ${ }^{1}$ ), bereits das erst 1802 bis 1805 erbaute Viewegsche haus ${ }^{2}$ ) steht. Die drei teile der einleitung, 'vorbericht', 'genealogie der herzöge von Braunschweig' und 'geschichte (in II 'kurze geschichte') von berzog Heinrich dem löwen ....., sind hier ebenso geordnet, wie in den vier obigen undatierten drucken des gedichts; auch finden wir den falschen geburtstag des herzogs Heinrich Julius, das falsche todesjahr Friedrich Ulrichs und das unrichtige geburtsjahr herzog Augusts wider. Ausserdem treten uns manche abweichungen, lutcken wie zusätze, sowol mit den oben genannten ausgaben des gedichts als auch bei diesen beiden prosaerzählungen unter einander entgegen. Auch die 'geschichte vom herzog Heinrich dem löwen auf seiner langen reise's) weicht von dem.inhalte des gedichtes nicht unwesent-

1) Dieser wie der holzschnitt des titelblattes sind in beiden ausgaben nach verschiedenen clichés desselben holzstockes hergestellt.

2) Vgl. H. Schröder und W. Assmann 'Die stadt Braunschweig' (Br. 1841) 8. 78.

3) In dem Einbecker drucke lautet die überschrift dieses ab- 
lich ab. Das wunderbare in Heinrichs erlebnissen ist be deutend abgeschwächt. So wird z. b. in dem Frankfurter drucke (I) der vertrag mit dem teufel als ein traum in einer anmerkung erzählt, im Einbeker (II) ganz fortgelassen, in beiden aber die ruckkehr des herzogs durch ein dahersegelndes schiff bewerkstelligt. Als bräutigam der herzogin wird in I ein prinz Cabixtus aus Schwaben genannt, dem nach der rückkehr des herzogs eine prinzessin Marianne aus Franken gegeben wird; in II fehlt dieser teil der erzählung gänzlich. Den 'anhang' (erzäblung aus Titius' exempelbuch etc.) haben sie wider gemeinsam. Den schluss macht in $I$ eine mit dem übrigen in keinem zusammenhange stehende erzählung: 'Ei so beiss!', in II ein gedicht: 'Die treue des löwen'.

Abgesehen von dieser sozusagen volkstlumlichen uberlieferung, welche wir so eben verfolgt haben, ist das gedicht aber auch in vollem wortlaute oder in prosaauflösung in einer anzahl von werken enthalten, welche auf mehr oder weniger wissenschaftlicher grundlage beruhen. Doch haben alle diese bearbeiter nur jüngere formen des gedichtes gekannt, keiner von ihnen bat den ursprünglichen druck Gödings benutzen können.

Reichard ${ }^{1}$ ) hat bei seiner prosaerzählung des inhalts des gedichts, welcher zahlreiche verse desselben eingefugt sind, offenbar einen jener undatierten nachdrucke der ausgabe von 1727 benutzt, da er s. 127 selbst sagt, dass sein gewährsmann die geschichte selbst bezweifelt und deswegen auf den ersten 10 seiten diesclbe nach der wahrheit beleuchtet. Das trifft bei jenen ausgaben vollkommen zu.

J. Görres nennt für die kurze prosawidergabe des gedichts in seinen 'Teutschen volksbüchern' (Heidelberg, 1807) s. 90-93 als quelle einen druck, der genau denselben titel fuhrt wie nr. 1 und 2 der nachdrucke der ausgabe von 1727.

Die brüder Grimm erzäblen im zweiten teile ihrer deutschen sagen (Berlin, 1818) s. 241-247 die sage von Heinrich

schnittes: 'erzählung von den abenteuern auf der reise des herzogs Heinrich des löwen'.

1) Vgl. Bibliothek der romane VIII B. (Riga, 1782) s. 125-136. 
dem löwen 'nach dem volkslied', ohne dass sich erkennen liesse, welche ausgabe des gedichtes ihnen vorgelegen habe.

J. G. Busching hat in seinen Volks-sagen, märchen und legenden (n. a. Leipzig, 1820) s. 211-242 das gedicht im wortlaute aufgenommen; ihm haben, wie or $\mathbf{s .} 450$ bemerkt, ein alterer und ein neuerer druck vorgelegen, wol ebenfalls solche der oben besprochenen bearbeitungen der ausgabe von 1727, mit deren texte jener in der hauptsache ganz thereinstimmt.

K. Simrock hat das gedicht im ersten bande seiner deutschen volksbücher (Frankf. a/M., H. L. Brönner 1845) s. 1-40 ebenfalls vollständig widergegeben. In der gestaltung des textes hat er sich zwar mancherlei freiheiten gestattet, im wesentlichen aber die fassung der volkslieder des vorigen jahrhunderts beibehalten. Das titelbild dieser ausgabe zeigt Heinrich den löwen vor einem turme stehend, an dem die schilde von Baiern, Sachsen und Braunschweig hangen; der lōwe liegt neben ihm. Ausserdem schmulicken das gedicht sieben andere holzschnitte, scenen der sage darstellend.

Daneben ist ein sonderabdruck dieser ausgabe erschienen mit folgendem titel:

Geschichte | des grossen Helden und Herzogen | Heinrich des Löwen | und seiner | wunderbaren hø̈chst gefährlichen Reise | [buchdruckerzeichen] | Frankfurt am Main | Druck und Verlag von H. L. Brönner. | Gedruckt in diesem Jahr.

In demselben wortlaute hat K. Simrock dann das gedicht ohne beigabe von bolzsebnitten in seinem werke 'Die geschichtlichen deutschen sagen aus dem munde des volks und deutscher dichter' (Frankfurt a. M., H. L. Brönner 1850) s. 278-304 nochmals herausgegeben.

In den siebziger jahren erschien: 'Heinrich der Löwe. Ein altes deutsches Volksbuch. Neu verfasst von L. Grote. Mit zwölf Holzschnitten. Hannover in diesem jahr. Im Selbstrerlage des Herausgebers'. Vorn auf dem umschlage steht eine nachbildung des titelbildes der Simrock'schen volksbucher. Auch von den anderen bildern ist ein teil nach den dort befindlichen holzschnitten gearbeitet, wenn nicht vielleicht beide auf eine gemeinsame vorlage zurluckgehen. Der text Grotes zeigt noch freiere behandlung als der Simrocks, welcher eben- 
falls benutzt ist. Eine zweite auflage des werkes ist im verlage von Trustav Jacob erschienen.

H. Pröhle hat seiner prosadarstellung der sage Heinrichs des löwen in der zweiten auflage seiner 'Deutschen sagen' (Berlin, 1879) s. 3-14, wie er s. 289 f. angibt, ausser dem 8. 292 erwähnten Einbecker drucke die s. 287 besprochene Wolfenbuttler handschrift $\mathrm{zu}$ grunde gelegt. $\mathrm{Lr}$ hat der letzteren folgend manche ausdrticke und wendungen des originals beibehalten, die allen anderen ausgaben und darstellungen bereits verloren gegangen waren.

Ein abdruck der dichtung Heinrich Gödings nach dem originaldrucke von 1585, der nachfolgt, wird hiernach den freunden deutscher sage und volkstumlicher dichtung gewiss nicht unwillkommen erscheinen.

Zuvor ist jedoch zum schluss der abhandlung noch darauf aufmerksam zu machen, dass unser verfasser des volksgedichts von Heinrich dem löwen nicht mit dem dichter Heinrich Göttings zu verwechseln ist, von welchem Goedeke in dem zweiten bande seines Grundrisses ${ }^{2}$ s. 285 zwei gedichte anfuhrt: 'Niemandt: Wie fast Jederman an jhm wil Ritter werden' etc. und 'Bewerte Kunst Goldt vnd Geldt zu machen. Erffordt, Georg Bawman 1590'. Denn letzterer hiess nicht, wie Goedeke ihn zwar nennt, Götting, sondern ganz zweifellos Göttingi d. i. Göttings. Er stammte ferner, wie der beiname Witzenhusanus zeigt, nicht aus Braunschweig, sondern aus Witzenhausen; er lebte 1585 zu Eifurt und war 1590 schulmeister zu Gebesee, einem städtchen im jetzigen regierungsbezirke Erfurt. Die vorrede des ersten schriftchens ist 'Erffurdt den 4. Februarij, Anno 1585' mit 'Henricus Göttingi, Witzenhusanus' unterschrieben; der titel des zweiten gibt als verfasser an: 'Henricum Göttingi Witzenhusanum Ludi Gebeseni M.', und die unterschrift des widmungsgedichtes lautet: 'Anno 1590. Heinricus Göttingi'. Danach ist es unmöglich, den maler Heinrich Göding und diesen dichter für ein und dieselbe person zu nehmen. 


\section{Eine schöne alte Histori | von einem Fürsten vnd Herrn Herrn| Hertzogen zu Braunschweig vnd Lüne- | burgk: In gesangs weis gerichtet, | Im 1585. Jahr. | \\ H. G.}

1. Man sagt von starcken Helden, sie sein zu preysen hoch; [A ii a] Darumb so mus ich melden von einem Herren auch: Er ist von Edlem Stamme vnd ist auch lobens werth, Von wegen grossen Thaten fuhrt er lillich das Schwerdt.

2. Preiss woldte er erlangen, zog weit in frembde Landt, Abenthewer zu erfahren, das kam jhm auch zu handt; Wagt derwegen leib vnd leben, wie jhr jetzt hören werd, Ja, wie man find beschrieben, er war ein Degen hart.

3. Er nam an Ritter vnd Graffen, der hochgeborne Fürst, Es waren sein Vnderthanen, nach Ehren jeden dürst. Sie kamen an ein Wasser: die Gäul liessen sie stahn Vnd seumbten sich nicht lange, zu Schiff theten sie gahn.

4. Das Schiff man fertig machte vnd lieffen schnell daruon, Sie fuhren Tag vnd Nachte, kein Landt sie traffen an.

Es zerbrachen jbre Segel, sie kamen da in noth;

Gros kummer stund jhn zu handen, jeder wündscht jhm den Todt.

5. Sie lagen da fast lange, die Speise hat ein endt. [A ii $^{\mathbf{b}}$ ]

Dem Herrn war fast bange, er hub auff seine Hendt:

Ach GOTT, thue dich erbarmen, wir müssen leiden den Todt, Kom du zu hülff vns armen, wir han weder Speiss noch Brodt.

6. Einer klagt dem andern den kummer, auch dieses gros Ellendt; Jeder war muth von hunger, sie wunden jhre Hendt.

Der Herre sprach mit sinnen: wir stehn alle warlich blos, Wir mögen nichts begitinnen, jeder mach auff sich ein Loss.

7. Die Loss wurden gemachet, wie man nun hören thut, Ein jeder darauff trachtet, man legt sie in ein Hutt;

Vnd ward gentzlich beschlossen, wer erstlich herausser kem, Sol sein gantz vnuerdrossen, sich den andern geben heim.

8. Das Loss fiele zum ersten auff einen kühnen Heldt. Er sprach balde von hertzen: machts, wie es euch gefeldt, Mein Leib wil ich euch geben dahin zu ewrer Speiss, Nembt mir als bald das Leben, theilt vnter euch mein Fleisch.

9. Ihr mögt mich brathen vnd sieden, ich gebs euch hertzlich gern, [A iii a] Allein wolst, GOTT, behüten vnsern frommen Landes Herrn. Es gehe gleich vber vns alle, wir sein klein oder gros, Ach GOTT, das ja nicht falle auff vnsern Herrn das Loss! 
10. Der Heldt warde geschlachtet, wie man das liesset noch; Speise man aus jhm machet, hunger war der beste Koch.

Er war balde vorzehret von seinen Mitgesellen;

Der hunger sie solches lehret. ein ander must sich einstellen.

11. Auff welchen das Loss thet fallen, thet sich einstellen gern. GO'T'I gab das glück für allen, er verschont jmmer den Herrn. Er stund mit einem Knechte, ware nechst GOTT sein trost; Gros waren sie in nöthen, der Herr war nicht erlost.

12. Der hunger hield nicht stille, er war bey' jhnen gros. Der Herr sprach: es ist mein wille, wir beyde werffen das Loss; Auff wehn es dann thut fallen, sol den andern verzehrn.

Der Knecht rieff laut mit schallen: ich thue solchs nicht meim Herrn.

13. Sie theten beyde lossen, der Knecht sahe das nicht gern. [A iii b] Das Loss da thete fallen auff sein getrewen Herrn.

Der Knecht solte jhn tödten, befahl der werde Mann;

Hoch waren sie in nöthen, der Knecht wolte nicht dran.

14. Der Kuecht sprache mit trewen: Herr, es ist als verlohrn. Last ewer Leben nicht gerewen, jhr seid ja hochgeborn.

Von Leder wil ich euch machen gar bald ein newen Sack, Ihr mögt des glücks erwarten, jhr seid noch Jungk vnd starck.

15. Der Knecht nain in der güte den thewren Helden werth, Nehet jhn in Ochssen heute vnd legt zu jhm sein Schwerdt. Ach thue es GOT'I erbarmen, wie stehe ich jetzt in noth! Meinen Herrn hab ich begraben vnd ist doch noch nicht todt.

16. $\mathrm{Zu}$ handt kame geflogen ein grimmiger Greiff gros, lst war vnd nicht erlogen, - hald nach dem Herren schoss, Fast jhn mit seinen Klawen, furth jhn bald in sein Nest, Der Herr thete sich frewen: GOTT thue bey mir das best!

17. Der Greiff floge von hinnen, mehr Speise er begert. [A iv a] Der Herr thet sich besinnen, er greiff sein scharffes Schwerdt, Er dancket GO'I'T dem HERREN vnd schneit sich aus der haut; Er sahe sich freudig vmme vnd fast ein guten muth.

18. Die Greiffen theten schreyen, sie begerten bald des Herrn. Ich sag es auff mein trewen, er thet sich jhrer erwehrn.

Er rieff zu GOtt dem HERREN, der half jhn ferner aus noth, Thet sich der Vogel erwehren vnd schlug sie alle zu todt.

19. Er steig gar bald herunder wol aus dem Greiffen Näst, Es nam den Herren wunder der vngehorten Gäst.

Man thut noch wol anschawen zu Braunschweig in der Burgk, Da hengt ein Greiffen Klawe, bracht er mit ohne sorg. 
20. Der Herre kam in weiten im $W$ ald, thet sich vmbschawen, Er sahe gar grimmig streiten einen Drachen mit dem Lawen. Er sprach: ich wil es wagen, sol ich gleich bleiben Todt, Thete auff den Drachen schlahen, sprach: das walde der liebe Gott!

21. Ich hab offt hören sagen, der Lew sey ein trewes Thier, [A $\nabla^{\mathrm{b}}$ ] Drumb wil ichs mit jhm wagen kegen dem Drachen vngehewr; Ich hoff, vns soll gelingen, der Drach sol liegen todt, Wil jhm dapffer beyspringen, dem Lewen helffen aus noth.

22. Sie theten da fast ringen, jeder sein sterck bewerth: Der Herr herzu thet springen mit seinem guten Schwerdt; Der Lewo ward das jnnen, fürm Drachen er nimmer weicht, Der Herr aus klihnen sinnen bald auff den Drachen streicht.

23. Der Drach spert auff sein Rachen gegen dem viel werden Mann. Der Herr begundt zu lachen, er sprach den Lewen an;

Der Lewe mit freiem muthe schnell auff den Drachen sprang, Der Drache schrey fast laute, das in dem Waldt erklang.

24. Der Herr mit freyem muthe schlug auff das wilde Thier Mit seinem Schwerdt so gate, aus jhm gieng wildes Fewr. Das sabe der Lew so gute, trewe er dem Herren both; Der Herr aus freyem muthe schlug da den Drachen todt.

25. Der Lewe thet sich legen zum Herrn vnd seinem Schildt, [B a] Er thete seiner pflegen, er fieng jhm Hirsch vnd Wildt; Grosse trew empfeht der Herre von diesem wilden Thier, Er thets jhm machen gare, sagt man, obn alles Fewr.

26. In diesem grossen Walde warn sie mit Wasser vmbgeben.

Er besann sich schnell vad balde: wie thue ich mit dem Lewen? Eine Hort thet er bald machen von Holtz vnd auch von Reiss; Der Lewe thet fleissig trachten, das er bekem ein Speiss.

27. Die Hort die war gebunden, er legt sie auff das Meer, Satzt sich darauff von stunden, sahe weit vmb sich daher.

Der Lewe kam gegangen, hat jhm ein Wildt gehatzt;

Der Herr seumbt sich nicht lange, het sich erst niedergesatzt.

28. Der Lewe trawret sehre, er fandt sein Herren nicht, Er lieff fast hin vnd here, sahe gar weit vmb sich, Er hort des Herren stimme, er sahe jhn auff dem Hort, Sprang bald aus grossem grimme zum Herren auff den Hort.

29. Sie flossen Tag vnd Nachte, wo sie der Windt hintrieb; [Bb] Der Herr auch jmmer wachte, für angst er wenig schlieff, Het auch viel lieber gesehen, der Lew wer blieben da. Wie sol vns nun geschehen? vnglücke kömpt vns nah. 
30. Ach G0'T'T, thue dich erbarmen, rieff er, hub auff die Hendt, Hilff doch zu Landt mir armen, die Speise hat ein endt.

Es war wol zu erbarmen, wie man erachten kan,

Der Lew an seinen Armen sahe jhn fast trawrig an.

31. Ja wunder mus ich sagen, wie man es offt erfindt; Mancher Feindt thut hass tragen, wenn er es enden kündt. Aber GOTT kann es wenden, mus kommen jhm zu gut, Sein Vnglücke auch enden vnd helffen aus der Noth.

32. Der Herr sich hart verwachet, het Tag vnd Nacht kein rub. Gar baldt sich zu jhm machet der Wirth aussm Nobiskrug:

New Zeitung wil ich dir eagen, bör wol auff meine wort, Du liegst in Wassers wagen, must endtlich sterben todt.

33. Zu Braunschweigk sein eingzogen, Ja gestern zu Mittag, - [B ii a] Ist war vnd nicht erlogen, was ich dir jetzundt sag, Man wird da Wirtschafft halten, ist jedem wol bekandt, Ein ander aus frembden Landen, der kriegt dein Weib vnd Landt.

34. Der Herre sass in trawren, er glaubt es were war: Ich bin ja aus gewesen lenger dann sieben Jabr, Sie werden nicht anders dencken, ich sey wegk von der Welt; Zu GOtt wil ich mich lencken, der machs wies jhm gefeldt.

35. Ja hör, ich wil dir sagen, du sagst noch viel von GOTT; Du liegst ins Wassers wagen, er hilfft dir nicht aus noth. Ich fuibr dich heut als balde zu dem Gemable dein, Auch zu dein Freunden allen, wenn du wilt mein eygen sein.

36. Sie hatten ein lang gespreche, der Herr wolt wilgen nicht: So ich mein gelubte breche gegen GOTT dem ewigen Liecht, Ob ich gleich bin ein Herre von Braunschweig hochgeborn, Fiel von Gott meinem Herren, so wer ich ewig verlorn.

37. Eins wil ich dir vorschlagen, gehe nur nicht lang zu rath: [B iib] Ich wil dich heut ohn Wagen führn gen Braunschweigk vor die Stadt, Ja one einigen schaden auffm Geyersbergk legen nieder;

Da solstu meiner warten, bis ich kom schier herwider.

38. Ich wil alsbald verschaffen den Lewen an das Orth, Vnd so ich dich findt schlaffen, - nun merck wol auff mein Wort: Alsdann solstu mein eygen in meinem Reiche sein.

Er wold jhn gern betriegen vmb Leib vnd Seele sein.

39. Ach GO'TT, thue mich erretten! wie thue ich nun der sachen? Ich wil gar trewlich beten, wil darzu fleissig wachen. Ach GOTT, thue mir bescheren heut einen seligen Tag! Ich befehl mich GO'TT dem HERREN, biss der Lewe kompt hernach. 
40. Der Herr thet sich besinnen, gab doch sein willen drein, Wie er möcht kommen von hinnen zu der allerliebsten sein: Ach GOtt, wolst mich bewahren diesen Tag vnd auch die Nacht, In Gottes geleyt zu fahren, ehe die Wirtschafft wird verbracht.

41. Er nam den werden Herren, fürt jhn in lïfften hin, [B iii a] Er meint, er sol sein werden, het einen guten gewyhn. Vor Braunschweigk leget er nieder den Edlen Herren frum: Ich kom gar baldt herwider; kanstu wol wachen nuhn?

42. Der Herre war fast müde, ist warlich nicht erlogen : Ach GO'TT, mich doch behüte, zufallen wolln mir die Ogen. Hilff GOTT, das ich nicht schlaffe, es möcht mir vbel gehn, Möcht kommen in sein Rachen, darzu in ewige Pein.

43. Er thet sich nieder lencken, der schlaff satzt jhm fast $\mathrm{zu}$; Er war nicht zu verdencken, hat lang gehat kein rhu; Er lag auffm Geyersberge zu Braunschweig für der Stadt, Wie man wol kan gedencken, von der Reyse war er math.

44. Es wehret nicht gar lange, Nobiswirth thet einher schweben Vnd het gar fest vmbfangen den frommen getrewen Lewen. Er sahe den Herrn liegen, er dacht er wer gar Todt. Er liegt dort an dem Berge, ist kommen da in noth.

45. Der Lewe thet laut schreyen, weil der Herr sich nicht rïhrt, $-\left[\mathrm{B}\right.$ iii $\left.{ }^{b}\right]$ Den 'T'euffel thets gerewen, das er jhn hat gefiihrt: Der Herr von solchem gschreye gar schnell vad bald erwacht; Da thets den 'Tenffel grewen, warff den Lewen, das er kracht.

46. Ja, so der Herr geschlaffen, wer kommen vmb Leib vnd Seel. Der lieb GO'TT thut solchs schaffen, von jhm kompt Leben vnd Heyl; Er hilfft in diesem Leben vnd endtlich aus dem l'odt, Thet seiner ferner pflegen, balff jhm aus dieser noth.

47. Der Herre fiel da nieder, er dancket Gott dem HERRN, Richt sich darnach anff wider; es wil nun Abendt werdn. Wer er den Tag nicht kommen, wer jhm ein grosser schad, Wie jhr jetzt habt vernommen, er kam fast eben spat.

48. In die Stadt kam er gegangen, der Lewe folgt jhm nach. kr war gar schlecht empfangen, nach der Burgk war jhm gach. Er hört ein gros gedöhne; er dacht: was mag das sein? Er thet sich balde lencken, nachm Hause stand sein sinn.

49. In sein Hauss wolt er schreyten, man wolt jhn nicht einlahn, [B iva] Trabanten vnd auch Leute die drawten jhn zu schlan.

Was wiltu allhier machen wol in des Fürsten Hoff? Wir sehens an dein sachen, du bist ein vmmelauff. 
50. Gros wunder nam den Herren, was er da sahe vnd host:

Es darff noch wol wahr werden, was der Teuffel mir sagt.

Was bedeut das gedöhn vnd pfeiffen? ist hier ein frembder Herr?

Gebt mir bericht, jhr Leute, was seins für newe Meer?

51. Der Herr ist ja nicht frembde, er ist vns wol bekandt;

Ich sag, das er bekïmmet heut das Brannschweigisch Landt

Mit vnser gnedigen Frawen; dann sie ist hochgebohrn,

Sie ist ein Witwe in trewen, jhren Herrn hat sie verlohrn.

52. Der Herr verwnndert sich sehre, thet eylen mit der Sacb, Er trath zu jhn anhere, thet freundlich bitten die Wach:

Hetten sie kein bedencken, solln thun den willen sein,

Er wolt nicht mehr begeren dann nur ein Becher Wein.

53. Der Herre bath fast sehre, er wolt gar nicht ablan; [Bivb]

Es war jhr Landes Herre; der Abendt gieng heran.

Er sprach zu einem in trewen: sprich doch die Fürstin an,

Es sol dich nicht gerewen, du scheinest ein trewer Mann.

54. Vnd thue sie freundlich bitten ein trunck von jhrem Wein, Den wolt sie herunder schicken; math ist das Hertze mein.

Er sahe an den Lewen vnd auch den werden Mann,

Er lieff gar schnell vnd balde zeigt es der Fürstin an.

55. Die Braut die thete lachen: was ist es für ein Mann?

Es warn jhr seltzame sachen, das er ein Lewen sol han.

Von Goldt gab sie ein Geschirre: las jhn den Trincken aus,

Er ist ein Abenthewer; wie kompt er in das Hauss?

56. Man trugk den Becher hienunder, er trancke aus den Wein.

Warlich mich nimmet wunder, woher du magest sein,

Das du begerst zu trincken allein von diesem Wein,

Den thut man allein einschencken der frommen Hertzogin.

57. Er nam ein Ringk von Golde, von ander war er geschlagen, [Ca]

In Becher warff er jhn balde, bath sehr, man wolt jhn tragen

Allein für die Fulrstin milde; darauff war geschnitten ein

Sein Helm vnd auch das Schilde; das trugk er jr hienein.

58. Der Knecht nam das Geschirre, er thet jhm nichts mehr sagen;

Es daucht jhn Abenthewre, fuir die Fürstin thet ers tragen.

Er sprach: zart gnedige Frawe, eine Fürstin hochgeborn,

Thut diss Ewer goad anschawen, haben sie diss Goldt verlorn?

59. Sie nam das Goldt zu handen, eygen thet sie es anschawen;

Ihr Hertze lag in banden; sie sahen all auff die Frawen.

Sie ward entferbet sebre, bald ward sie wie ein Leich,

Sie gedacht: ist es mein Herre, der Hertzog von Braunschweig? 
60. Die Braut stund auff mit eyle, bald in die Kammer gieng. In einer kleinen weile rieff sie den Kämmerling, Sprach: habet jhr geseben drunden den frembden Mann? Ja der für vnserm Hause sol mit dem Lewen stahn?

61. Er sprach: zarth gnedig Frawe, ich hab jhn wol gesehn, [C Thet jhn gar wol anschawen, der Lew thet mit jhm gehn; Der Lew ist jhm getrewe vnd ist jhm vnderthan; Viel Leut thun jhn anschawen, ist warlich ein feiner Mann.

62. Sie legt sich an die Zynnen vnd thet hienunder schawen, Sie ward jhres Herrn jnnen, er sass da mit dem Lawen. Hilff G0tt, das mir gelinge! was er mir hat geschickt, Ist von meins Herren Ringe. gar offt sie jhn anblickt.

63. Last jhn herauffer kommen, wir wolln jhn freundlich fragen, Wo er den Ringk genommen; er mus es warlich sagen. Den Ringk ken ich gar eben; mein Herr hat mir jhn geben, Da er von mir thet scheiden. ach Gott, wer er bey leben!

64. That jhn von ander schneiden, das ist gewiss vnd war, $\mathrm{Da}$ er von mir thet scheiden lenger dann sieben Jahr. Kom ich dann nu nicht wider auff dieses Hauss vnd Saal, Sprach da mein Edler Herre, so nembt ein ander Gemahl.

65. Jederman nam es wunder: was wil daraus noch werdn? [C iia] Die Räht namen besonder den frommen Landes Herrn. Sie fragten $\mathrm{jhn}$ in trewen vmb diese wunder ding, Ja wo ers het genommen, es wer jhres Herrn Ringk.

66. Der Herr begundt zu lachen, sprach: es wird werden gut. $\mathrm{Ja}$ fleissig thet er trachten, ob er möcbt sehn die Braut. Von keim bab ich bekommen, das aag ich euch furwar, Ich hab jhn hie genommen lenger dann sieben Jahr.

67. Sie theten jhn anschawen: er war ein ernster Mann. Einer lieff zu der Frawen, that jhr solchs zeigen ahn: Der Ringk der were kommen ja an sein rechtes Orth, Er het jhn wider geleget, dahin er billich ghort.

68. Die Fürstin wundert sich sehre, gieng schnell durch einen Saal, Sie sprach: ach Gott, mein Herre ist es, mein liebes Gemahl; Denn der Ringk ist gewesen dem liebsten Herren mein. Ach mucht er sein genesen, wer noch beim leben sein!

69. Sie thet den Herrn anschawen, für frenden fiel sie nieder. [ $\mathrm{C}$ ii b] Der Herr sahe an die Frawen, thet $j$ hr aufthelffen wider.

Es nam wunder alle Herren, sie sprachen all zugleich:

Was wil da noch aus werden? hilff GOTT von Himmelreich. 
70. Die Fürstin thet jhn kennen, both jhm jhr weisse Handt. Ach Herr, jhr wolt euch nennen: seid jhr der Herr im Land? Ihr solt euch vns anmelden, sagen wir zu dieser stund;

Wir preysen GOtt dem HErren, das jhr seid kommen gesundt.

71. Vor zeiten war ich ein Herre, sage es ohn allen spott, Mir geschicht jetzt wenig Ehre, mus es befehlen GOT'I';

Ich war ein Hertzog ohn sorgen, das sag ich euch fiirwar, Zu Braunschweig ausgezogen, ist lenger dann sieben Jahr.

72. Seid jhr des Lands ein Herre, seid vns all Gott wilkomn! Sie theten jhm gros Ehre, dann er war mildt vnd fromb. Die Fürstin fiel danieder, sie danckt dem lieben GOT $\Gamma$ : Mein Herr ist kommen wider, GOTT hat jhn wol behut.

73. Zu Tisch thet man jhn weisen, jederman es gerne hort, [C iii a] Man thet jhn besser speisen, als jhm geschach auff der Hort, Zur Braut satzt man jhn nieder, jederman thet jhn anschawen; Man thet auch wol versorgen sein getrewes 'Thier den Lawen.

74. Was sol man jmmer sagen? dem Breutgam kam die meer, Er war wol zu beklagen, das eben kam der Herr:

Nun ist meine sach verloren, durchn Korb bin ich hindurch; Wer ich noch höher gebohren, stehe jetzundt fast in sorg.

75. Der Breutgam trawret sehre, es thet jhm weh der hohn. Wens nicht wer der Lands Herre, er wolt daruon nicht lohn. Nach der Braut stund sein verlangen: ich hab ein Wildt geiagt, Ein ander hat es gefangen, das sey ja Gott geklagt.

76. Die Herrn giengen zusammen, man pfleget guten rath;

Der Furst thet zu jhn kommen, ja jeden er da bath, Guten rath solten sie geben; in gnaden wirds erkandt:

Ja weil er bet sein leben vnd Herre wer im Landt.

77. Seiner gnad sie theten dancken, die Sach wer nicht verlorn: [C iii b] Ein Frewlein ist hier ans Francken, ist warlich hochgebohrn; Dem Breutgam wolln wir sie geben, das sol geschen zu handt, Sie ist fast seaberlichen als eine in diesem Landt.

78. Dem Fürsten thet gefallen, die Vohrschläg waren gut; Er lacht, das es thet schallen, gar frölich war sein muth. Gehet bald in der eyle, zeigt es dem Herren an:

Er sol doch Wirtschaffit halten, das Frewlein sol er han.

79. Die Herrn eyleten sehre, giengen $20 \mathrm{jhm}$ ins Gmach, Sie sagten jhm die mehre, erzehlten jhm die Sach:

Zeittung wolln sie jhm bringen, die Sach wolt werden gat, Man wolt jhm bald zufüren ein schöne Junge Braut. 
80. Ewer gnad haben vernommen von vnserm Landes Herrn, Das er ist wider kommen aus frembden Landen fern. GOT'T hat jhn auch bewahret aus vieler angst vnd noth; Wir waren all in trawren, als wer er langest todt.

81. Weil es dann so GOTT schaffet, die euch zur Ehe vermahlt, [C iva] Die Fürstin auch darauff hoffet, vnd es ja nicht sein solt, Euch ein aus hohen Stammen ein Frewlein ausserkorn. Diesen Raht hat gegeben vnser Fürst hochgeborn.

82. Der Herre sprach mit sitten: Ihr trewen Räht vnd Herrn, Eines wil ich euch bitıen, kündt jhr mir das gewehrn, Das vnser gnediger Herre gebe sein Willen drein;

Ich sag es auff mein trewe, sol mir nu die liebste sein.

83. Spürt jhr den gnedigen Willen von denen hochgebohrn, So gehet in der stille zu der jhr habt erkohrn.

Ich sag es euch fürwahre: mein eygen sol sie sein, Mit mir sol sie heim fahren gar baldt in meine Heimbt.

84. Sie seumbten sich nicht lange, giengen zum Frewelein; Sie wurden schon empfangen, hies sie wilkommen sein.

Mit züchtigem geberde drugen sie an jhr Sach;

Das Frewlein wehrt sich sehre, doch endtlich sie des lacht.

85. Sie wolte auffschub nemen, die Herren wolten nicht. [C iv b] Ein wenig thet sie sich schemen, gab doch hoflich bericht: Sie wolt fragen ihren Herren, darzu seiner gnaden Gemahl; Was sie jhr rathen theten, solt jhr gefallen wol.

86. Sie sprachen: Edel Jungfrewlein, vnser hohe Oberkeit Han geben jhren willen drein; gebt vns kurtzen bescheidt. Ja vnser Gnedige Frawe het jhn selber genommen, Wir sagens auff vnser trewe, wer er nicht wider kommen.

87. Nu Gottes will geschehe! wil ers dann also han, Sol ich nun greiffen zur Ehe, zeigts vnserm Herren an. Sprachen: das mlissen wir sagen, das vnser gnediger Herr Euch hat diss vohrgesehlagen, reicht euch zu grosser Ehr.

88. Das Frewlein gab jhren willen, sie hatte Ja gesagt. Sie zeigens an dem Herren, sprachen: jhr habt noch wol geiagt; Das Frewlein ist ewer eygen. GOTT gebe glück darzu! Wollen sie euch bald zulegen, solt haben gute Ruh.

89. Der Herr mit freyem muthe thet dancken diesen Herrn: [Da] Mein sache wird fast gute, nu bestehe ich noch mit ehrn. Sol ich nu Wirtschafft halten mit meiner newen Braut, Gott der HERR thue es walten, mein sach ist worden gut. 
90. Sie antworten in der eyle: es sol noch heut geschehn. Man nam da nicht viel weile, giengen bald zum Frewelein. Man nam sie bey den handen vnd fürt sie zu dem Herrn; Er thet sie wol empfangen, er nam sie hertzlich gern.

91. Grossn danck thet er jhn sagen, thet sie empfangen fein Auff einem gïlden Wagen fubrt sie zur Kirchen nein. Ein jeder kundt nicht hören allda sein eygen wort Von Pfeiffen vnd Drommeten, desgleichen man nie erhort.

92. Man gab sie da zusammen, wie das noch offt geschicht. Da sie herausser kamen, war alles zugericht. Jederman war in freuden, die sach deuchtet jhn gut; Es war wol zugerichtet, doch nicht auff diese Braut.

93. Man thete Wirtschafft balten, man sahe da manchenMann [D ${ }^{b}$ ] Von Jungen vad den Alten gerüst bald auff dem Plan; Mit Rennen vnd 'Thornieren brach mancher seinen Spiess, $\mathrm{Ja}$, wie man hat gesehen, that jeder grossen fleis.

94. Die Wirtschafft hat ein ende, ein jeder Vrlaub nam, Gaben dem Herrn die Hende Braut vnd der Breutigam. Man thet sie auch geleiten, gros Gutt man jhn mit gab; Jederman so es gesehen, hat lust vnd frende darah.

95. Der Herre sass in Ehren, Regieret Leut vnd Landt; Man thete jhn fast ehren, leyst den frommen offt beystandt. Man thete jhn fast lieben den Herren zu Braunschweig Von wegen seiner trewe, macht manchen Armen Reich.

96. Biss in sein alten tagen thet jhn G0tt wol bewahrn, Sein Gemahl ohn alles klagen für vngelücke sparn. $\mathrm{Ja}$, die nach seinem Todo diss Landt theten regiern, Gab jhnen G0'TT aus güte viel Frewlein vnd Junge Herrn.

97. Der Herr leget sich nieder, von Alter war er schwach: [D ii a] Kom ich dann nicht auff wieder, so befehl ich meine Sach Christus meim lieben HERREN befehl ich Leib vnd Seel, Der wolle nun mein pflegen; von jhm kümpt leben vnd heyl.

98. Sein Gemahl weinet sehre, der Herr thet sie gesegnen: Mein bleiben ist nicht mehre, GO'T' wolt nun ewer pflegen, GOTT wolle euch bewahren, darzu auch Leut vnd Landt. Wol in derselben stunden both jedem da die Handt.

99. Er thet sein Geist auffgeben, der Edle Herre werth, Vnd endet da sein leben. man legt za jhn sein Schwerdt, Man thet ehrlich begraben ja dieses Fürsten Leib;

Das Grab wird noch gesehen im Thume zu Braunschweigk. 
100. Jederman trawret sehre vmb den Herrn bochgebohrn, Desgleichen dass wilde Thiere, hat auch sein Herrn verlorn.

Der Lewe legt sich nieder wol auff seins Herren Grab, Daruon wolt er nicht wider, bis er sein Geist auff gab.

101. Man thet die Ehr dem Lewen, man grub jhn für die Burgk; [D ii b] Man thut das Grab anschawen, wer jetzt noch gehet durch.

Ein Lewe ist gegossen, teglich thut man jhn sehn, Stelt fast ein guten possen; auffer Seule thut er stehn.

102. Ein Greiffenklaw thut hengen vber dieses Fürsten Grab, Er thete sie mit bringen, wie ich berichtet hab;

Dabey ist nu zu mercken, das solches ist geschehn.

Ia mitten in der Kirchen wird diss Grab noch gesehn.

103. Ach Gott, du wolst bewahren all fromme Oberkeit In diesen schweren Jahren für angst vnd grossem leidt, Gnediglich sie behiiten allhie in diesem leben, Das sie von Landt vid Leuten gute Rechnung mügen geben.

104. Von wegen der Geschichten hab ich dis Lied gedicht, In eyle thet ichs machen, hets sunst besser gericht, Dem Fürsten Stamm zll Ehren, auch meinem Vaterlandt, Befehl ich GOTT dem HERREN in seine starcke Handt.

\section{Anmerkungen.}

Der vorstehende abdruck gibt im ganzen den originaldruck in möglichster genauigkeit wider. Er unterscheidet sich von ihm nur durch das absetzen der verse, die einfuhrung der interpunktion und die abänderung einzelner angenscheinlich durch druckfehler in den text geratener worte, welche sich in folgenden verzeichnet finden. Die reime des dichters sind sehr ungenau, ja sio fehlen zumal am ende der ersten halbzeilen nicht selten ganz. Es finden sich hier z. b. worte wie:

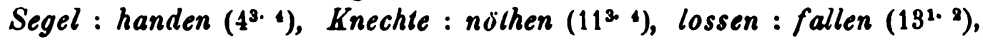

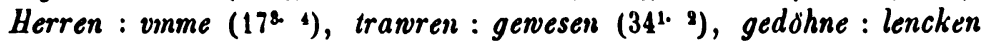
(48. 4), frembde : bekómmet $\left(51^{1 \cdot 2}\right)$ u. 8. w. zusammengestellt. Streng innegehalten hat der dichter den klingenden ausgang in der mitte der langzeilen. Nur in 5 versen ist er nicht uberliefert, aber durch ganz leichte änderangen herzustellen, die demnach gewiss nicht unberechtigt erscheinen.1) Auch der stumpfe reim am ende der langzeilen findet sich fast durchgehends gewahrt und zu seiner aufrechterhaltung hat der dichter auch kürzungen nicht verschmäht, wie verzehrn : Herrn (123. 4),

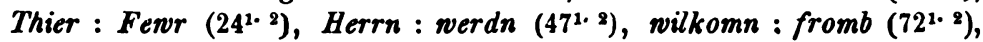

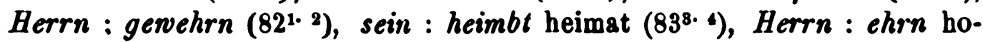
nores (89.2). Ich habe daher an den stellen, wo das eine reimwort

1) An vier stellen $\left(17^{8} .39^{4} \cdot 69^{3} \cdot 85^{8}\right)$ ist Herrr statt Herren zu schreiben und $74^{8 .} 4$ verlorn: gebohrn statt verloren: gebohren. 
die kiirzere form zeigte, auch bei dem zweiten eine solche eingesetzt.') Dasselbe verfahren anch an den 18 stellen einzuschlagen, wo beide reimworte die längere form aufweisen ${ }^{2}$ ), schien mir eine zu grosse abweichung von dem überlieferten texte zu sein, obwol es keinem zweifel unterliegen kann, dass auch diese versausgänge als stumpf gemeint anzusehen sind.

64. Das auslosen der schiffsinsassen in der hungersnot und das verzehren der vom lose getroffenen ist ein zug, der uns in den verwanten fassungen, bei $M$. Wyssenhere ${ }^{3}$ ) und im niederländischen volksliede ${ }^{4}$ ), nicht begegnet.

73. geschlossen drack.

74. dem druck.

173. HERRN druck.

18. erwehren druck, vgl. str. 123. 4. 821. 2. 891. 2.

193. Vgl. iber die greifenklaue, welche friber im dome zu Brannschweig uber dem grabe Heinrichs des löwen hieng und noch jetzt in jener kirche gezeigt wird, str. 102 und das auf s. 286 und 290 gesagte. Mich. Wyssenhere erwähnt (str. 30) mehrere klauen, die der fuirst den jungen greifen abgeschlagen habe und die hangen zu brüneczwigk in der stat. Im niederlïndischen volksliede findet sich nichts entsprechendes.

201. in weiten anf weite, freie strecken, anf lichtungen im walde. Spätere ausgaben haben den ausdruck nicht mehr verstanden, sie schreiben: der Herr that sich von weiten im Walde ziemlich umschaun.

21. der] des druck.

21. vngehewer druck.

254. Fewer druck, vgl. str. 241. .

294. nahe druck.

821. ruhe druck.

32. Der Wirth aussm Nobiskrug, wie str. $44^{1}$ der Nobiswirth, bedeutet den teufel. Die bearbeitung von 1727 nennt daher statt jene

1) Das letzte e ist gestrichen in den durch den drack Uberlieferten formen: erwehren $\left(18^{2}\right)$, vngehewer $\left(21^{2}\right)$, Fewer $\left(25^{4}\right)$, nahe $\left(29^{4}\right)$, ruhe $\left(32^{1}\right)$, werden $\left(65^{1}\right)$, Ruhe $\left(8^{4}\right)$, sparen $\left(96^{2}\right)$.

2) Mitgesellen : einstellen (103.4), vmbschawen : Lawen (201.2), vmbgeben : Leven (26. $\left.{ }^{2}\right)$, nieder : herwider (373. 4), sacken : wachen (391. $\left.{ }^{2}\right)$,

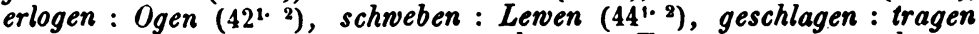
$\left(57^{1 \cdot 2}\right)$, sagen : tragen $\left(53^{1 \cdot 2}\right)$, anschawen : Frawen $\left(59^{1 \cdot y}\right)$, schawer,

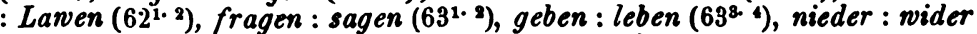
$\left(69^{1 \cdot 2}\right)$, schaven : Lawen $\left(73^{3 .} 4\right)$, genommen : kommen $\left(86^{3 \cdot}\right)$, gesegnen : pflegen (981. 2), leben : geben (1038. 4).

8) Uas gedicht Michael Wyssenheres ist gedruckt in Massmanns 'Denkmaelern deutscher sprache und literstur' (Munchen etc., 1828) 8. $122-137$.

4) Von dem niederländischen volksliede benutze ich einen druck, den K. Koppmann noch 1873 anf der strasse von Gent, wo er feil geboten wurde, gekauft und stadtarchivar Hänselmann in Braunschweig mir freundlichst zur verfugung gestellt hat.

Beiträge zur geschichte der deutschen sprache. XIII. 
2usdrücke zu gebrauchen geradezu den Teufel und den Satan (vgl. s. 289). Ueber den ursprung des wortes nobis oder des ursprïnglichen obis aus abyssus (hölle) oder wol richtiger ans abd. opasa und opisa, got. ubizwa, mhd. obese, mnd. ovese, womit die vorhalle, das dach, die dachtranfe and weiter die grenze bezeichnet wurden, uber die bedeutung des Nobiskruges und Nobishauses als wirtshauses der unterwelt oder des teufels, uiber die bezeichnung von schenken an den grenzen und an begräbnisstätten als Nobiskrüge vergl. Grimms Deutsche mythologies s. $953 \mathrm{fff}$; Brem.-niedersächs. wtb. III s. 254; Gödeke in der Ztschr. d. histor. ver. f. Niedersachsen, jahrg. 1852 s. 367 f.; Schiller und Lübben, Mnd. wtb. III s. 190; Casper im Korrespondenzbl. d. ver. f. niederd. sprachf., V. jahrg. s. 28 und dazu ebendas. VI. jahrg. s. 19, insbeenondere aber den aufsatz Ludwig Laistners in der Germania hg. v. Bartsch (1881) N. R. XIV (XXVI) s. 65-95 u. 176-199. Noch deutlicher sind mythische anklänge an der betr. stelle bei $\mathrm{M}$. Wyssenhere str. $66 \mathrm{ff}$. Hier kommt der fürst

vnder das Woden her, dae die bössen geiste ir wonüg han;

einer dieser geister bewirkt in ähnlicher weise wie bei Göding die rückkehr des helden. Im niederländischen volksliede tut dies der satan.

334. Ein ander aus frembden Landen ist der bräutigam der herzogin; in späteren bearbeitungen erscheint ein Fürst aus fremden Landen, in der ganz jungen prosaauflösung ein Prinz Cabixtus aus Schwaben. In dem gedichte M. Wyssenheres wird der bräutigam allgemein ein man oder ähnlich (str. 68 und 94) genannt. Dass es sich um einen dienstmann des fürsten handelt, geht aus der anrede desselben an ihn hervor, str. 95:

\section{Blib by mir vor als nach Als eyn getrüner dinsiman.}

Aber es wird keineswegs gesagt, dass es derjenige gewesen, dessen schutze der fürst str. 9 gemahlin nnd herrschaft ubertragen hat. Das niederl. volkslied bezeichnet den bräutigam der herzogin nicht niiher, G. Thym macht ihn in seinem gedichte von 'Thedel von Wallmoden v. 558 zum pfalzgrafen.

34. Dass der herzog 7 jahre fortgewesen, wird widerholt gesagt, so noch $64^{2} .66^{4}$ und $71^{4}$. Die zeitbestimmung fehlt bei M. Wyssenhere, findet sich aber im niederl. volksliede, wie in dem verwanten Möringerliede (Uhlands Volkslieder 8. 7i6). Bei Thym ist Heinrich der luwe bei seiner begegnung mit Thedel von Wallmoden, welcher ihm die bevorstehende heirat seiner gattin meldet, zwei jahre von der heimat fort.

354. alle druck.

37. Der Geyersbergk, eine verhochdentschung des noch jetzt gebräuchlichen niederdeutschen ausdrucks Giersberg, bildet eine mässige anhohe, welche damals nahe bei der stadt Braunschweig im osten derselben lag, jetzt aber in diẹ neuen strassenzlige der erweiterten stadt 
anfgenommen ist. Vgl. str. $43^{3}$. Bei M. Wyssenhere (str. 74) wird der fürst für syn burgk niedergesetzt.

394. HERRN druck.

422. Die nd. form Ogen ist wol nur durch flïchtigkeit des in Niederdeutschland erwachsenen dichters in den text geraten. Der reim zwang keineswegs dazu; es finden sich z. b. hoch : auch (11. 2), Hoff : vmmelauff (49. ${ }^{3}$ ) verbunden.

45'. Der löwe schreit, weil er denkt, dass sein herr gestorben sei. Diese schöne begründung für das geschrei des löwen nnd das erwachen seines herrn findet sich nur hier; sie fehlt bei M. Wyssenhere (str. 76) nnd im niederländischen volksliede.

484. Es ist, wie auch $49^{1}$, nur von dem Hause des herzogs die rede. Erst die bearbeitung von 1727 setzte dafür die bezeichnung Mosthauss ein (8. 290), die vollkommen richtig ist. Denn Mosthaus ist aus nd. moshus, mhd. muoshiss cænaculum, speisesasl entstanden und man bezeichnete damit den pallas, den saalbau in der burg Heinrichs des löwen zu Braunschweig, der zur zeit eine würdige widerherstellung erfährt. Vgl. L. Winter, Die burg Dankwarderode zu Brannschweig (Brsunschw. 1883) 8. 40 ff.

57: Das zerbrechen des ringes beim abschiede des helden zum zweck der widererkennung kommt schon in der frühsten uns tiberlieferten gestalt der sage von Heinrich dem löwen vor, im Reinfried von Braunschweig ( $\mathbf{} .14762 \mathrm{ff}$.), wo die rückkehr selbsi allerdings nicht mehr erzählt wird, da die handschrift des gedichts leider weit fruher bereits abbricht. Ebenso findet sich dieser zug bei $M$. Wyssenhere str. 10 und im niederländischen volksliede; im letzteren bricht jedoch nicht der herzog, sondern die herzogin den ring.

65'. werden druck, vgl. str. 471.2.

69. Herrn druck.

694. weren druck.

743. verlorn drack. - durchn Korb bin ich hindurch, vgl. über diese redensart sowie uber die jetzt gebräuchliche einem einen Korb geben die mit reichen belegstellen versehenen susflubungen $\mathbf{R}$. Hilde. brands in Grimms Deutschem wtbche B. V (Leipzig 1873) sp. $1800 \mathrm{ff}$. 'Ursprünglich ist diess der korb durch den ein liebender des nachts zum fenster aufgezogen werden sollte und der im fall der abweisung von der höhe fallen gelassen oder zum durchbrechen des bodens eingerichtet wurde, dass der liebende durchfallen musste.' Aus der sitte entwickelte sich ein symbol. Später 'schickte man dem abzuweisenden bewerber einen korb ohne boden'.

744. gebohrn druck.

772. Das fräulein, welches der bräutigam der herzogin zar entschädigung erhält, wird nur im allgemeinen aus Francken genannt; erst die späte prosaauflösung fügt einen namen Princessin Marianne ein. Die vermählung des bräutigams mit einer anderen braut nach der rückkehr des fürsten fehlt bei M. Wyssenhere und im niederl. volksliede, 
findet sich dagegen beim Möringer, welcher dem nebenbuhler seine tochter zar frau gibt (Ubland 2. 2. 0. 8. 783).

81. die] sie druck.

85. ihren Herren] meinen Herrn druck.

88. Ruhe druck.

962. sparen druck.

1013. Der eherne lowe auf dem burgplatze zu Braunschweig, den herzog Heinrich schon vor seiner fahrt ins heilige land im jabre 1166 hat aufstellen lassen. Vgl. die angabe in den Stader Annalen (Mon. Germ. Hist. XVI, 345); Durre, Geschichte der stadt Brannschweig (Br. 1861) s. 66. Die errichtung des denkmals erzählt ebenfalls M. Wyssenhere (str. 98); im niederl. volksliede lässt die herzogin dssselbe aufstellen. 1024. Das grab herzog Heinrichs des lowen und seiner gemshlin Mathilde liegt inmitten des domes zu Brannschweig. Ueber die herrlichen steinbilder, die anf demselben ruhen, vgl. Bethmann Westermanns monatshefte aug. 1881 a. a. o. s. 554 ff., sonst K. Steinmann, Die grabstätten der fürsten des Welfenhauses (Braunschweig 1885) в. 20 ff. 